\title{
Endothelial-to-mesenchymal transition in human idiopathic dilated cardiomyopathy
}

\author{
YEQING XIE $^{1,2^{*}}$, JIANQUAN LIAO $^{1 *}$, YONG YU $^{1}$, QI GUO $^{1}$, YINGZHEN YANG $^{1}$, \\ JUNBO GE $^{1}$, HAOZHU CHEN ${ }^{1}$ and RUIZHEN CHEN ${ }^{1}$ \\ ${ }^{1}$ Key Laboratory of Viral Heart Diseases, Ministry of Public Health, Shanghai Institute of Cardiovascular Diseases; \\ ${ }^{2}$ Division of Nephrology, Zhongshan Hospital, Fudan University, Shanghai 200032, P.R. China
}

Received March 29, 2017; Accepted October 9, 2017

DOI: $10.3892 / \mathrm{mmr} .2017 .8013$

\begin{abstract}
Dilated cardiomyopathy (DCM) is characterized by left ventricular dilation and cardiac fibrosis. Emerging evidence indicated that endothelial-to-mesenchymal transition (Endo-MT) is a crucial event during organ fibrosis. This study was performed to clarify whether Endo-MT contributed to the progression of cardiac fibrosis in DCM. Cardiac samples from patients with DCM and control were obtained. The presence of endothelial markers, cluster of differentiation (CD)31 and vascular endothelial (VE)-cadherin, and mesenchymal markers, $\alpha$ smooth muscle actin (SMA) and fibroblast-specific protein 1 (FSP1) was performed using immunohistochemistry. Co-localization of endothelial markers and mesenchymal markers were identified using confocal immunofluorescence staining. Serum procollagen type I carboxy-terminal propeptide (PICP) and procollagen type III amino-terminal propeptide (PIIINP) were measured by ELISA. Protein levels of Wnt, $\beta$-catenin and Snail were determined using western blot analysis. Immunohistochemistry and double-immunofluorescence staining demonstrated that the expression of CD31 and VE-cadherin were significantly decreased in DCM samples, whereas the FSP-1, and $\alpha \mathrm{SMA}$ were significantly increased. CD31 and VE-cadherin labeling indexes were respectively negatively correlated with left ventricular end-diastolic diameter (LVEDD) (CD31 r=-0.82, $\mathrm{P}<0.01$; VE-cadherin $\mathrm{r}=-0.73, \mathrm{P}<0.01$ ), while FSP-1 and $\alpha$ SMA were positively associated with $\operatorname{LVEDD}(\alpha$ SMA $r=0.65, \mathrm{P}<0.01$, FSP1 $r=0.53, \mathrm{P}<0.01)$ and left ventricular ejection fraction $(\alpha$ SMA r=-0.18, P<0.05; FSP1 r=-0.21, P<0.05). Furthermore,
\end{abstract}

Correspondence to: Ruizhen Chen, Key Laboratory of Viral Heart Diseases, Ministry of Public Health, Shanghai Institute of Cardiovascular Diseases, Zhongshan Hospital, Fudan University, Shanghai 200032, P.R. China

E-mail: chen.ruizhen@zs-hospital.sh.cn

*Contributed equally

Key words: dilated cardiomyopathy, endothelial-to-mesenchymal transition, cardiac fibrosis, Wnt signaling, myocardial remodeling
PICP and PIIINP levels were positively associated with the co-expression labeling indexes (CD31/SMA co-labeling index and PICP r=0.727, $\mathrm{P}<0.01$; CD31/SMA co-labeling index and PIIINP $\mathrm{r}=0.741, \mathrm{P}<0.01$; VE-Cadherin/FSP-1 co-labeling index and PICP $\mathrm{r}=0.716, \mathrm{P}<0.01 ; \mathrm{VE}$-cadherin/FSP-1 co-labeling index and PIIINP $\mathrm{r}=0.648, \mathrm{P}<0.05)$. Western blot analysis indicated that proteins levels of Wnt signaling and snail were significantly increased in DCM samples. These results suggested that Endo-MT is potentially implicated in the pathogenesis of myocardial fibrosis and remodeling during the development of DCM, indicating a potential therapeutic target for DCM treatment.

\section{Introduction}

Dilated cardiomyopathy (DCM) is the third most common cause of heart failure, which is characterized by ventricular chamber enlargement and systolic dysfunction (1). Myocardial fibrosis is a pathological entity of extracellular matrix remodeling, often leading to increased myocardial stiffness, which may promote cardiac dysfunction (2). Both reactive (interstitial and perivascular) fibrosis and reparative (replacement) fibrosis are found in DCM, indicating that inflammation and microvascular injuries were involved in the disease process (3). Cardiac fibrosis is associated with an adverse prognosis and impaired response to therapeutic interventions in selected groups of patients with DCM (4), indicating that anti-fibrotic therapies might be useful in improving cardiac function of the diseased heart. However, it was limited by an indistinct understanding of the origin of fibroblasts in the heart.

Fibroblasts are regarded as a predominant cellular mediator of fibrosis in the heart, which play an important role in regulating normal cardiac function and adverse myocardial remodeling (5). A subset of cardiac fibroblasts is of endothelial origin via a cellular transition which is referred to as endothelial-to-mesenchymal transition (Endo-MT) (6). Endo-MT is a process by which endothelial cells disaggregate, change shapes and migrate into the surrounding tissue, accompanied by loss of endothelial cell markers like CD31 and vascular endothelial cadherin (VE-cadherin), and up-regulation of mesenchymal markers like smooth muscle actin ( $\alpha$ SMA) and fibroblast specific protein-1 (FSP1). It was first described in embryonic heart development, where endocardium cells loss 
its expression and become the mesenchymal cells that form the matrix of atrioventricular cushion and give rise to cardiac valve and septa (7). A plethora of evidence indicated that Endo-MT mediated the development of fibrosis in multiple diseases, including cardiac infarction (8), hyperglycemia (9-10), chronic pulmonary hypertension (11), tumor progression (12-13) and kidney injury (14). Compelling evidence reveal that endothelial cells, as a subset origin of cardiac fibroblasts, were involved in the progression of cardiac fibrosis via Endo-MT (6).

Given that endothelial cells were reported to contribute to pool of organ myofibroblasts, Endo-MT was investigated to explore its potential role in the progression of DCM in the present study.

\section{Materials and methods}

Patient population. Forty consecutive patients with idiopathic dilated cardiomyopathy with heart failure (mean age $50.3 \pm 11.6$ years, $30 \mathrm{M} / 10 \mathrm{~F}$ ) were recruited for the study. Patients with abnormal renal and hepatic functions were excluded, as endothelial functions were affected by renal and liver diseases. Patients with ischemic cardiomyopathy were not included in the study to eliminate other possible concomitant factors in the endothelial dysfunction of cardiac tissue. Left ventricular samples were obtained from these DCM patients for heart transplantation. Control heart samples were collected at forensic autopsy of road traffic accident fatality from ten persons of corresponding to age and sex ( 7 males and 3 females, mean age $42.7 \pm 20.5 y$ ), excluding cardiovascular, liver and kidney diseases. The present study conformed to the principles outlined in the Declaration of Helsinki and procurement of tissue for research. The study protocol was approved by the Ethic Committee of Zhongshan Hospital affiliated to Fudan University.

Histopathological analysis. The excised heart specimens were fixed with $10 \%$ phosphate buffered solution (PBS) formalin and embedded in paraffin. Sections (4- $\mu$ m-thick) were stained with hematoxylin-eosin (HE) for the measurement of the myofibrils distribution. Deposition of collagen was analyzed by Sirius Red staining (SR). Each sample slice was photographed (HE x200 magnification; SR x40 magnification) under the microscope (Olympus BX51; Olympus, Tokyo, Japan). All photos were analyzed with image-Pro Plus 6.0 analyzing software (Media Cybernetics, Bethesda, MD, USA) by computer.

Detection of serum procollagen type I carboxy-terminal propeptide $(P I C P)$ and procollagen type III amino-terminal propeptide (PIIINP). PICP and PIIINP were measured from blood samples collected from DCM patients before surgery and healthy subjects. All samples were centrifuged immediately at $2,000 \mathrm{rpm}$ for $10 \mathrm{~min}$ and store at $-70^{\circ} \mathrm{C}$ until assay. PICP and PIIINP were measured by enzymelinked immunosorbent assay (ELISA) (E90570Hu and E90963Hu; USCN Life Sciences Inc., China).

Immunohistochemistry. Cardiac specimens were fixed with $10 \%$ PBS formalin, and 4-um-thick paraffin-embedded tissue sections were prepared. Immunostaining was performed using primary antibodies against FSP-1 (ab27957, rabbit polyclonal;
Abcam, Cambridge, UK), aSMA (ab5694, rabbit polyclonal; Abcam), CD31 (ab24950, mouse polyclonal; Abcam), VE-cadherin (ab7047, mouse polyclonal; Abcam) according to standard protocols. To block the activity of endogenous peroxidase, sections were immersed in $0.3 \%$ hydrogen peroxidase in methanol for $20 \mathrm{~min}$ at room temperature. After pretreatment with blocking goat serum, sections were incubated overnight at $4^{\circ} \mathrm{C}$ with individual primary antibodies as FSP (1:100), aSMA (1:100) CD31 $(1: 1,000)$ and VE-cadherin (1:50). Then sections were incubated with secondary antibodies conjugated to peroxidase-labeled polymer, and then were washed and developed using diaminobenzidine (DAB) as the chromogen. Brown yellow staining of cytoplasmic membrane was considered positive binding. Semi-quantitative assessment of overall positive area was performed on randomly chosen high-power fields (HPF) in each section with a Leica-Qwin multipurpose color image processor (Leica, Germany).

Double immunofluorescence staining. The co-expression of endothelial markers and mesenchymal markers were detected using confocal fluorescence microscopy. Frozen cardiac sections were cut into $5 \mu \mathrm{m}$ thick sections, fixed in phosphate-buffered paraformaldehyde (PFA) at room temperature for $30 \mathrm{~min}$, and then permeabilised with $1 \%$ Triton X-100 in PBS for $5 \mathrm{~min}$. After treated with $5 \%$ BSA for $1 \mathrm{~h}$, the tissues were then incubated with two primary antibodies at $4^{\circ} \mathrm{C}$ overnight. The primary antibodys were $\alpha$ SMA (ab5694, rabbit polyclonal; Abcam) and CD31 antibody (ab24950, mouse polyclonal; Abcam), as well as FSP-1 (ab27957, rabbit polyclonal; Abcam) and VE-cadherin antibody (ab7047, mouse polyclonal; Abcam). Tissues were incubated with a mixture of two secondary antibodies that had been raised in different species and conjugated to different fluochromes (i.e., rhod red-conjugated goat-anti-rabbit and FITC-conjugated goat-anti-mouse; Jackson ImmunoResearch Labs, West Grove, $\mathrm{PA}, \mathrm{USA}$ ), in $1 \% \mathrm{BSA}$ for $1 \mathrm{~h}$ at room temperature in the dark, normal rabbit IgG was used for control. After the nuclei were stained with DAPI, slides were mounted with mounted with Mowiol antifade reagent (Sigma-Aldrich, St. Louis, MO, USA). Confocal images were captured using Leica TCS-SP5 laser-scanning confocal microscope. Digital images were analyzed with LAS AF software.

Western blot analysis. Western blot analysis was performed as previously described. Briefly, tissues were homogenized in RIPA lysis buffer supplemented with a protease and phosphatase inhibitor cocktail (1:100; Thermo Fisher Scientific, Waltham, MA, USA). Protein samples were separated by $10 \sim 12 \%$ SDS-PAGE and transferred to polyvinylidense difluoride membrane $(0.22 \mu \mathrm{M}$, Immobilin-P, Millipore, Billerica, MA, USA). Membranes were incubated with primary antibodies specific for Wnt (BS1777, Rabbit polyclonal, 1:500; Bioworld Technology, Inc., St. Louis Park, MN, USA), $\beta$-catenin (BS3730, Rabbit polyclonal, 1:500; Bioworld), and snail (BS1853, Rabbit polyclonal, 1:500; Bioworld), followed by incubation with horseradish peroxidase-conjugated secondary antibodies. Proteins were visualized using the Supersignal West Pico Chemiluminescence detection system (Pierce Chemical Co., Rockford, IL, USA). The arbitrary units were normalized to GAPDH and expressed as fold induction over control values. 
Statistical analysis. Data were expressed as the mean \pm SEM. Statistical significance was determined using the Student $t$ test and the Pearson correlation test. $\mathrm{P}<0.05$ was considered to indicate a statistically significant difference.

\section{Results}

Clinical characteristics of the study subjects. Clinical and echocardiographic features of patient population were summarized in Table I. Cardiac enlargement and impaired cardiac dysfunction were observed in DCM patients (mean LA chamber: $44 \pm 9 \mathrm{~mm}$; LVEDD $65.8 \pm 9.7 \mathrm{~mm}$; LVEF $28.5 \pm 8.3 \%$; BNP $780.4 \pm 308.9 \mathrm{pg} / \mathrm{ml}$ ). Most patients had premature ventricular contractions, among which five patients were implanted with pacemaker (ICD, implantable cardioverter-defibrillator) and two patients had both atrial fibrillation and premature ventricular beat. Over $80 \%$ patients were treated with $\beta$-blockers, whereas only $32.5 \%$ patients with ACE inhibitors and $47.5 \%$ patients with ARB.

Histopathological features and serum PICP and PIIINP levels. HE staining revealed moderate-to-severe myofibrils disarrangement, myocytes degeneration, interstitial vacuolization and replacement of fibrosis with or without inflammatory infiltrates in myocardial tissue samples of DCM patients. It was observed that interstitial fibrosis was markedly increased in pathologic cardiac samples after Sirius red staining (Fig. 1A).

PIIINP is an extension peptide of procollagen type III, which is cleaved off during conversion from type III procollagen to type III collagen. Elevated serum PIIINP is believed to reflect enhanced collagen turnover. In the present study, serum concentration of PICP was higher in DCM patients than in the healthy persons (mean value: $37.66 \mathrm{pg} / \mathrm{ml}$ vs. $32.03 \mathrm{pg} / \mathrm{ml}, \mathrm{P}<0.05$ ). Moreover, the PIIINP level was significantly increased compared to healthy group (mean value: $85.95 \mathrm{pg} / \mathrm{ml}$ vs. $60.06 \mathrm{pg} / \mathrm{ml}, \mathrm{P}<0.01$ ) (Fig. 1B). The concentrations of serum PICP and PIIINP represent the synthesis of type I and III myocardial collagen respectively. The higher serum PICP and PIIINP levels of DCM group indicated increasing collagen production, which was consistent with the pathologic results.

Endo-MT in DCM. Sections of 40 patients and 10 donors were stained respectively with endothelial markers and mesenchymal markers. In agreement with previous studies $(6,9,10)$, CD31 and VE-cadherin were used as markers of endothelial cells, and FSP- 1 and $\alpha$ SMA were used as markers of myofibroblasts cells in this study. Immunohistochemistry staining depicted that the expression of CD31 and VE-cadherin was significantly decreased in the DCM samples, compared with control (Fig. 2A). It was also found that no FSP-1-positive staining cells were detected in cardiac microvascular endothelial cells of normal healthy samples, whereas increased FSP-1-positive cells were found in the cardiac micrangium wall of DCM (Fig. 2A, P<0.01). Similar results were observed using $\alpha$ SMA staining (Fig. 2A, $\mathrm{P}<0.01$ ). Moreover, immunohistochemistry was performed for CD31, VE-cadherin, FSP-1 and $\alpha$ SMA. Compared with control, semiquantitative analysis indicated that the CD31 and VE-cadherin labeling indexes were significantly decreased in DCM samples $(C D 31, \mathrm{P}<0.01$;
VE-cadherin, $\mathrm{P}<0.01$ ), whereas the FSP-1 and $\alpha$ SMA labeling indexes were significantly increased (Fig. 2B, $\mathrm{P}<0.01$ ).

To further delineate Endo-MT, co-localization of endothelial markers and mesenchymal markers was analyzed using double immunofluorescence staining. An analysis of aSMA/CD31 and FSP-1/VE-cadherin demonstrated that some cells acquired both endothelial and mesenchymal markers, which occurred exclusively in cardiac sections of DCM patients, whereas no such cells was detected in normal cardiac samples (Fig. 3).

Correlation between Endo-MT and cardiac function indexes. As depicted in Fig. 4, CD31 and VE-cadherin immunestaining labeling indexes were respectively negatively correlated with left ventricular end-diastolic diameter (Fig. 4A, CD31 $\mathrm{r}=-0.82, \mathrm{P}<0.01$; VE-cadherin $\mathrm{r}=-0.73, \mathrm{P}<0.01$ ), while FSP-1 and $\alpha \mathrm{SMA}$ immunestaining labeling indexes were positively associated with left ventricular chamber enlargement (Fig. 4B, $\alpha$ SMA r=0.65, P<0.01, FSP1 r=0.53, P<0.01) and left ventricular ejection fraction (Fig. 4D, $\alpha$ SMA $r=-0.18$, $\mathrm{P}<0.05$; FSP1 $\mathrm{r}=-0.21, \mathrm{P}<0.05)$. However, neither CD31 nor VE-cadherin had statistically significant correlation with left ventricular ejection fraction (Fig. 4C). Moreover, neither left ventricular posterior wall (LVPW) nor intervetricular septum (IS) had statistically significant correlation with the CD31 or VE-cadherin indexes.

In addition, similar semiquantitative analysis was also performed for co-localization of endothelial markers and mesenchymal markers; and the CD31/SMA and VE-cadherin/FSP-1 co-labeling indexes were indentified. The fibroblasts coexpressed both the VE-cadherin and the FSP1 varied from 16 to $39 \%$, while the CD31/SMA co-localization index was $20-46 \%$. As depicted in Fig. 5, both circulating PICP and PIIINP levels were positively related with the co-expression labeling indexes (Fig. 5A: CD31/SMA co-labeling index and PICP $\mathrm{r}=0.727$, $\mathrm{P}<0.01$; Fig. 5B: CD31/SMA co-labeling index and PIIINP $\mathrm{r}=0.741, \mathrm{P}<0.01$; Fig. 5C: CD31/SMA co-labeling index and PICP $\mathrm{r}=0.727, \mathrm{P}<0.01$; VE-Cadherin/FSP-1 co-labeling index and PICP r=0.716, P<0.01; Fig. 5D: VE-cadherin/FSP-1 co-labeling index and PIIINP $\mathrm{r}=0.648, \mathrm{P}<0.05$ ). These results indicated that Endo-MT was probably associated with myocardial fibrosis and remodeling in the development of DCM.

Elevation of canonical Wnt signaling family members. Canonical Wnt/ $\beta$-catenin signaling pathway is an important regulatory mechanism in embryonic cardiac progress $(15,16)$. To identify signaling pathway and transcription factors that interact with the Endo-MT in human samples, we evaluated the Wnt signaling pathway and transcription factor snail. In this study, western blot analysis depicted that protein levels of Wnt and $\beta$-catenin were hardly detected in normal heart controls. However, these proteins were significantly increased in DCM samples compared with control (Fig. 6), suggesting that canonical Wnt/ $\beta$-catenin pathway was activated in DCM samples. Snail is well known for its capability to trigger Endo-MT. Our results demonstrated that snail was significantly elevated in DCM samples compared with control, suggesting that snail probably participated in the regulation of Endo-MT for mesenchymal markers induction. 
Table I. Characteristic of patient population (DCM, $\mathrm{n}=40$ ).

\begin{tabular}{lclc}
\hline Age (years) & $50.3 \pm 11.6$ & \multicolumn{1}{c}{ Sex $(\mathrm{M} / \mathrm{F})$} & $30 / 10$ \\
\hline NYHA class (\%) & & Atrial fibrillation, n (\%) & $8(25)$ \\
I & $0(0)$ & Premature ventricular contractions, $\mathrm{n}(\%)$ & $36(90)$ \\
II & $0(0)$ & Atrioventricular block, $(\%)$ & $3(7.5)$ \\
III & $26(65)$ & Pacemaker implantation, $\mathrm{n}(\%)$ & $5(12.5)$ \\
IV & $14(35)$ & Drug, $\mathrm{n}(\%)$ & $32(80)$ \\
Echo results & & $\beta$-blockers & $13(32.5)$ \\
left atrium (mm) & $44 \pm 9$ & ACE inhibitors & $19(47.5)$ \\
LV end-diastolic diameter $(\mathrm{mm})$ & $65.8 \pm 9.7$ & ARBs & $14(35)$ \\
LV end-systolic diameter $(\mathrm{mm})$ & $49 \pm 10.2$ & CCBs & $23(57.5)$ \\
LV ejection fraction $(\%)$ & $28.5 \pm 8.3$ & Digoxin & $30(75)$ \\
LV posterior wall $(\mathrm{mm})$ & $8.52 \pm 0.76$ & Diuretics & $780.4 \pm 308.9$ \\
Intervetricular septum $(\mathrm{mm})$ & $8.75 \pm 0.94$ & BNP $(\mathrm{pg} / \mathrm{ml})$ & \\
\hline
\end{tabular}

A
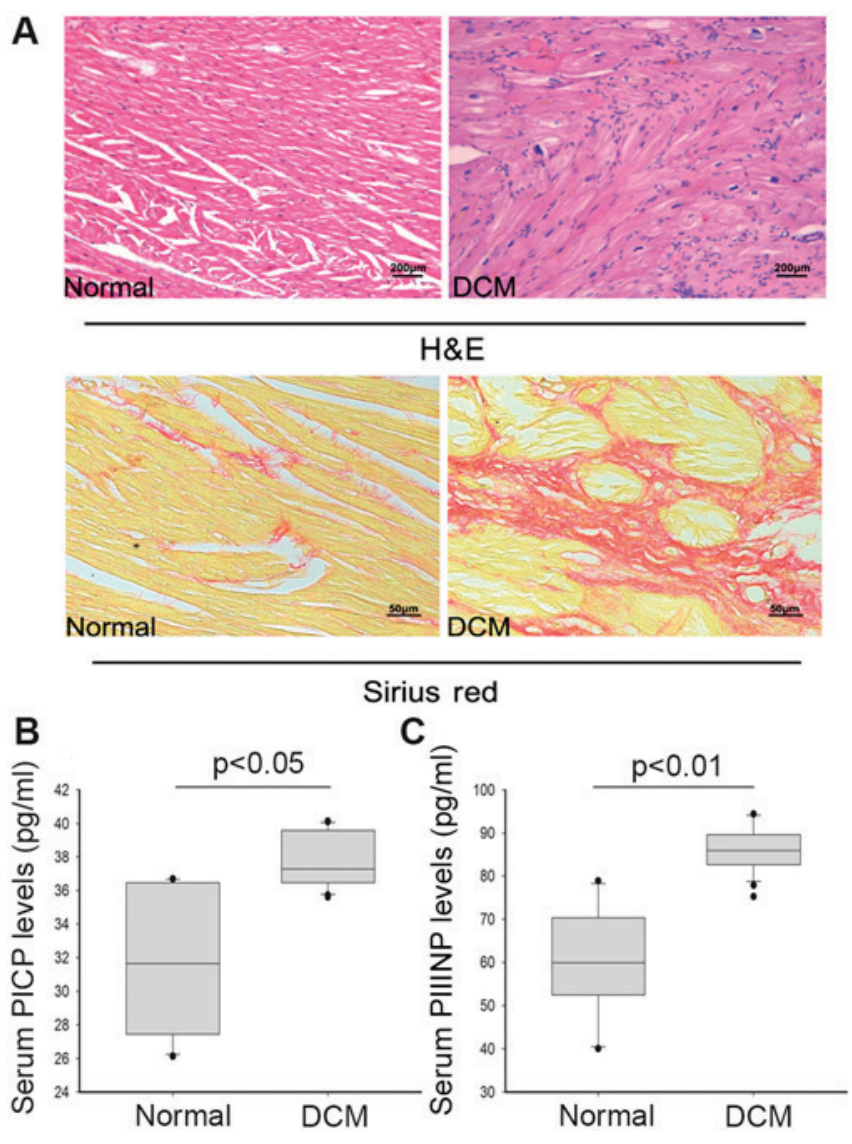

Figure 1. Cardiac fibrosis and remodeling in recruit patients: (A) Pathological features of enrolled sample: Increased amount of interstitial fibrosis was observed in DCM samples comparing control as indicated by HE and Sirius Red staining. (B) Serum PICP and (C) PIIIINP levels were significantly increased in DCM patient comparing control $(\mathrm{P}<0.05)$.

\section{Discussion}

The salient findings of this study indicated that Endo-MT might contribute to myofibroblasts recruitment in human DCM, characterized by decreased endothelial markers and increased mesenchymal markers. These markers also were correlated with cardiac function indexes and circulating levels of collagen I and III peptides. Moreover, our data revealed that canonical Wnt signaling pathway was involved in Endo-MT during the development of DCM.

Endo-MT was characterized by loss of endothelial cell markers and up-regulation of mesenchymal markers. It is now clear that the Endo-MT can also postnatally occur in various pathological settings, including cardiac fibrosis. Cardiac fibrosis and myocardial remodeling was the important pathologic features of DCM. However, the mechanism of cardiac remodeling has not been previously addressed. In this study, our results revealed co-expression of endothelial markers and mesenchymal markers in DCM cardiac samples, indicating that the accumulation of myofibroblasts originated from endothelial cells was probably implicated in myocardial fibrosis in DCM. Intriguingly, we found that both endothelial and mesenchymal markers were strongly correlated with left ventricular chamber diameter, whereas only mesenchymal markers indexes had their low correlation with LVEF.

The rate of extracellular synthesis of collagen type I can be assessed by measuring the serum concentration of PICP, which was formed during the extracellular processing of procollagen type I before collagen molecules form fibers. Moreover, PIIINP was formed during the extracellular conversion of procollagen type III into mature fibril-forming collagen type III. The predominance of the synthesis of collagen types I and III over their degradation results in the accumulation within the myocardium of an excess of collagen type I and type III fibers that characterizes fibrosis (17). As a marker of collagen type I synthesis, serum PICP was regarded as a marker of myocardial fibrosis in hypertensive heart disease (18). In this study, our result indicated that serum PICP and PIIINP was significantly elevated in DCM patients. Moreover, co-expression labeling indexes were respectively closely associated with PCIP and PIIIINP, which respectively presented collagen I and III synthesis and indicated increasing collagen production. These results indicated that increased serum PICP and PIIINP was probably associated with Endo-MT induced cardiac fibrosis in 

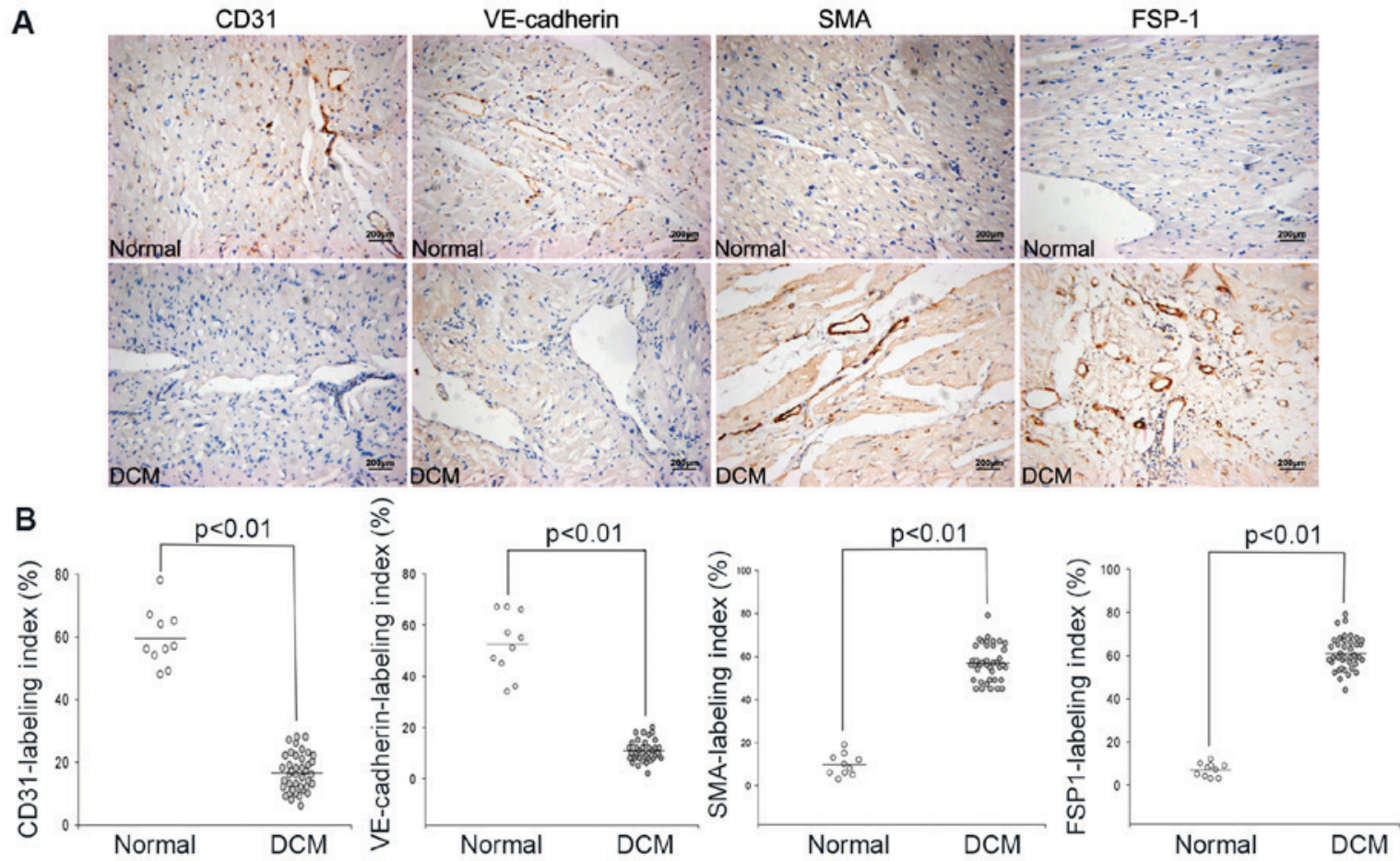

Figure 2. (A) Representative image and (B) immunostaining of endothelial and mesenchymal markers in both normal and pathologic cardiac sections: Compared with control, the expression of $\alpha$ SMA and FSP1 was increased in DCM specimen, whereas expression of CD31 and VE-cadherin was reduced $(\mathrm{P}<0.05)$.
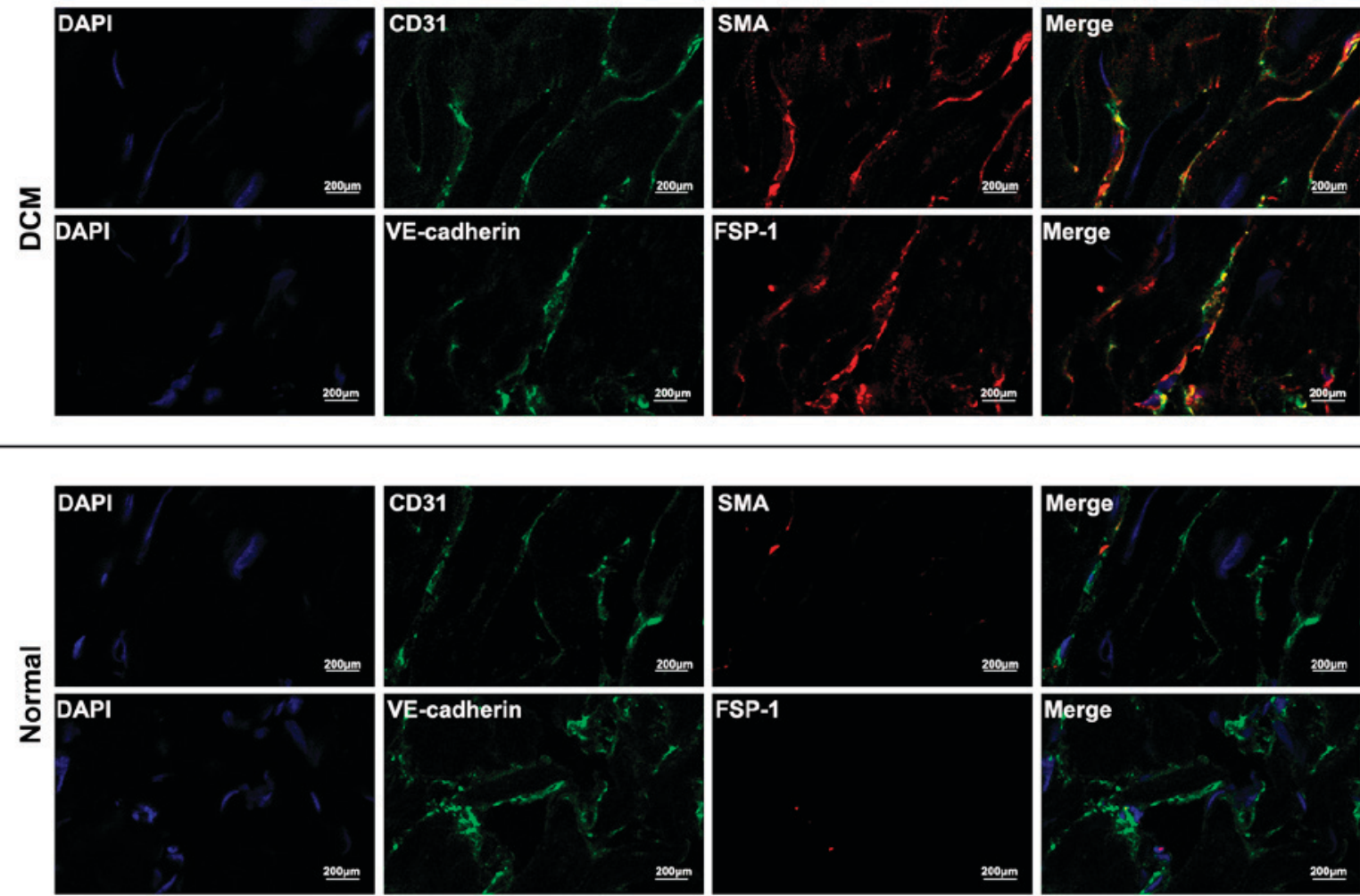

Figure 3. Co-localization of endothelial and mesenchumal markers in human cardiac samples: Double inmmunofluorescence staining of CD31 and $\alpha$ SMA, and VE-cadherin and FSP1 was performed for normal heart and DCM cardiac samples. The expression of CD31 and VE-cadherin were colored with green, and the expression of aSMA and FSP1 were colored with red. Nuclei were stained with 4'6-diamidino-2-phenylindole (DAPI). Coexpression of CD31 and $\alpha$ SMA was observed in cardiac endothelial cells of DCM, as well as VE-cadherin and FSP1.

DCM. Together, our results suggested that Endo-MT was associated with myocardial fibrosis, remodeling and cardiac dysfunction in pathogenesis of DCM, which was similar with the previous animal data that Endo-MT contributed to the progression of cardiac fibrosis in pressure overload-induced and diabetic-induced mice models $(6,8,19,20)$. The excessive 

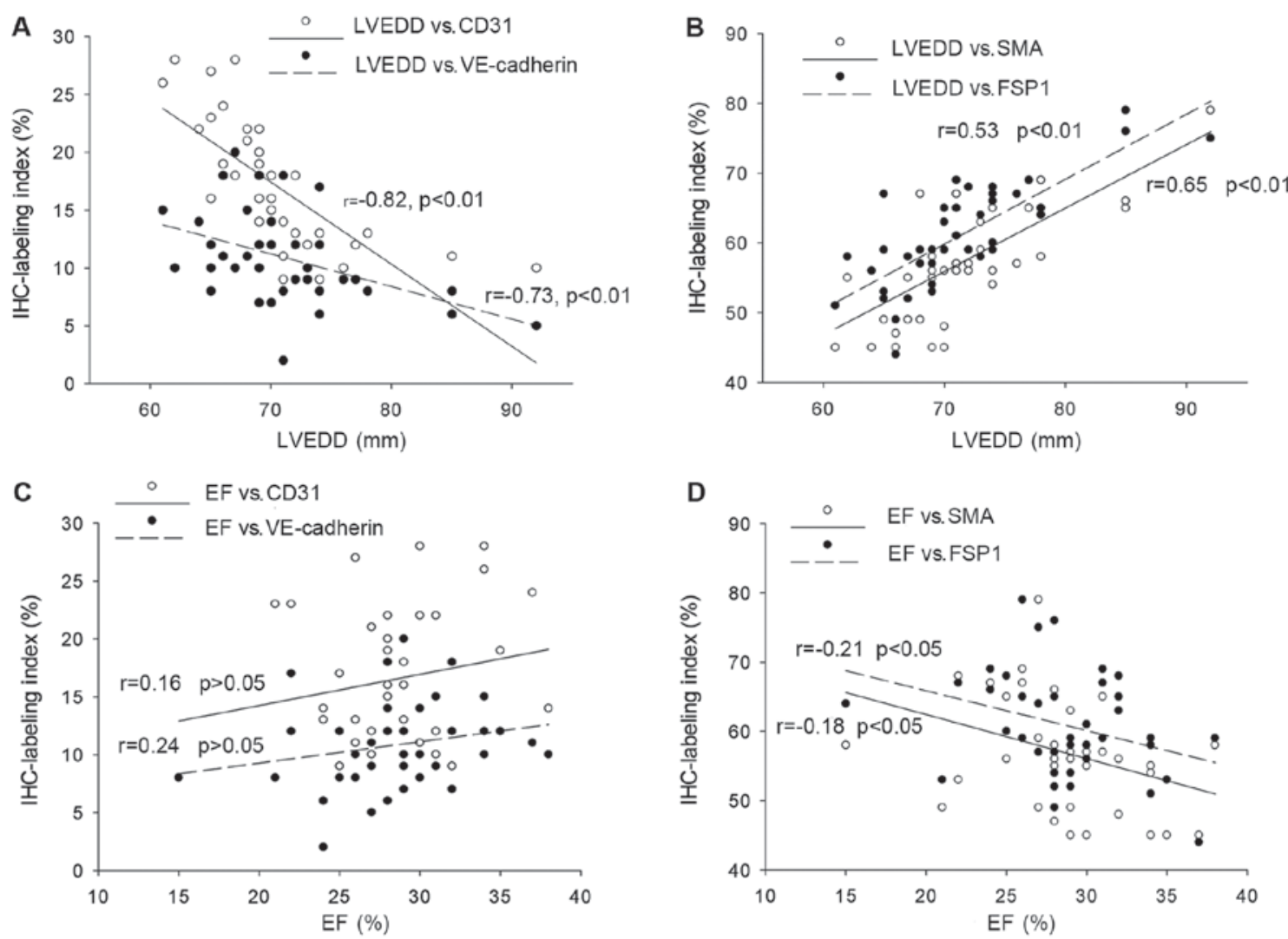

Figure 4. Relationship between cardiac functions and immunostaining indexes of endothelial and mesenchymal markers in DCM patients: Cardiac functions were demonstrated as left ventricular end diastolic diameters (LVEDD) and left ventricular ejection fraction (EF). CD31 and VE-cadherin immunestaining labeling indexes were respectively negatively correlated with left ventricular end-diastolic diameter (A) CD31 $\mathrm{r}=-0.82, \mathrm{P}<0.01$; VE-cadherin $\mathrm{r}=-0.73, \mathrm{P}<0.01$, while FSP-1 and $\alpha$ SMA immunestaining labeling indexes were positively associated with left ventricular chamber enlargement (B) $\alpha$ SMA $r=0.65, \mathrm{P}<0.01$, FSP1 $r=0.53, \mathrm{P}<0.01$ and left ventricular ejection fraction (D) $\alpha$ SMA $r=-0.18, \mathrm{P}<0.05$; FSP1 $\mathrm{r}=-0.21, \mathrm{P}<0.05$. However, neither CD31 nor VE-cadherin has statistically significant correlation with left ventricular ejection fraction (C) CD31 r=0.16, VE-cadherin $\mathrm{r}=0.24, \mathrm{P}>0.05$.
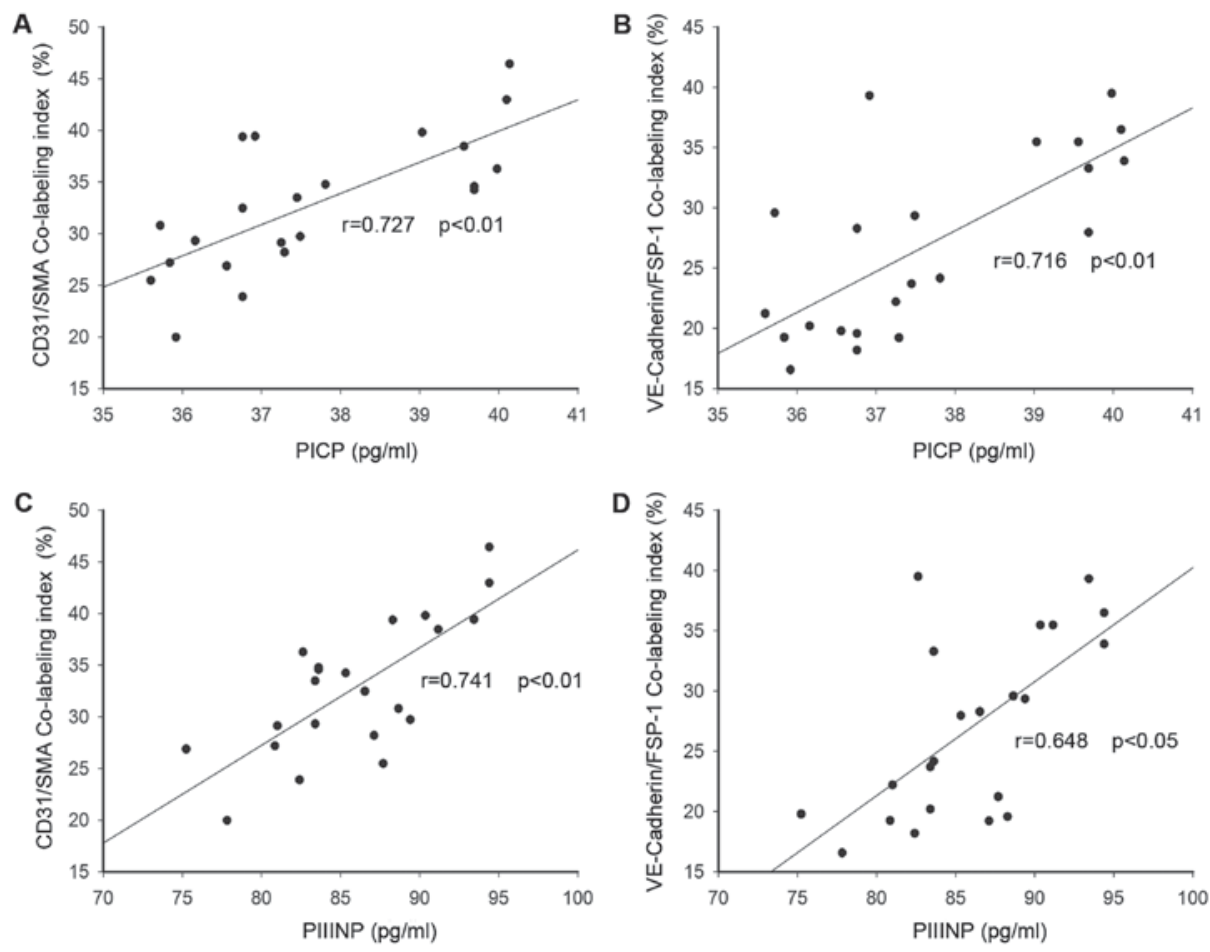

Figure 5. Relationship between serum levels of PICP and PIIINP and co-localization labeling indexes in DCM patients: Both circulating PICP and PIIINP levels were positively related with the co-expression labeling indexes. (A) CD31/SMA co-labeling index and PICP r=0.727, P<0.01; (B) CD31/SMA co-labeling index and PIIINP r=0.741, P<0.01; (C) CD31/SMA co-labeling index and PICP r=0.727, P<0.01; VE-Cadherin/FSP-1 co-labeling index and PICP $r=0.716$, $\mathrm{P}<0.01$; (D) VE-cadherin/FSP-1 co-labeling index and PIIINP $\mathrm{r}=0.648, \mathrm{P}<0.05$ ). 

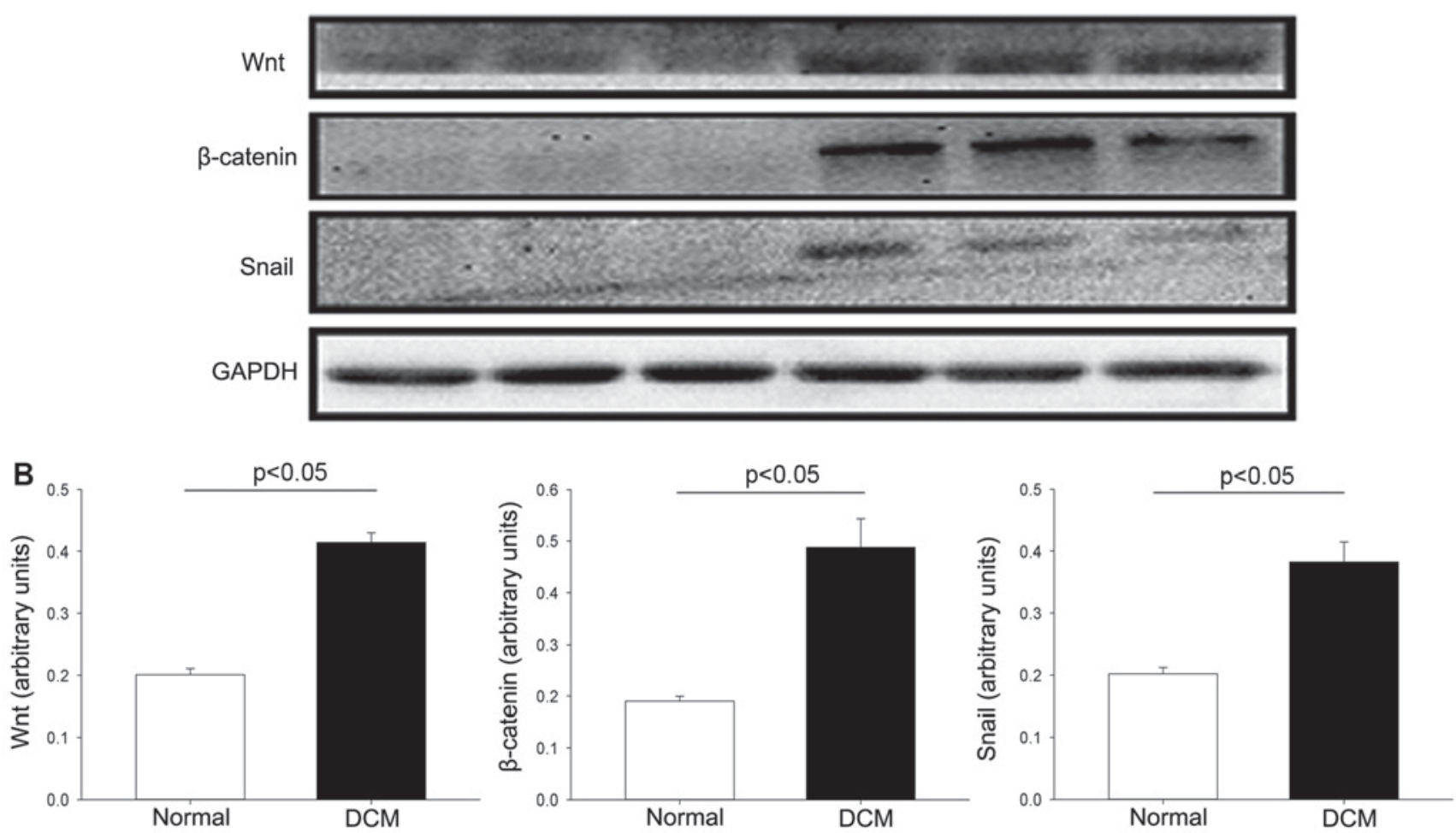

Figure 6. Wnt signaling pathway in DCM: (A) Western blot analysis for the expression of Wnt signaling proteins was performed on protein isolated from hearts of normal donors and DCM patients. (B) Quantification of proteins showed that conical Wnt-related protein was significantly increased in DCM specimen, compared with control $(\mathrm{P}<0.05)$.

formation of fibrotic tissue in the heart in turn reduces cardiac muscle tissue, thereby decreasing cardiac function (21).

Endo-MT has been extensively studied in generation of cardiac valve and endocardial cushion during embryonic heart development $(7,22)$, which provide a useful framework for guiding research on the Endo-MT in the postnatal pathologic diseases. Wnt family is highly conserved during embryogenesis (23) and required for adult tissue maintenance (24). Accumulating evidence indicated that Wnt signaling is required for different aspects of cardiac development during embryonic period, including myocardial specification, cardiac morphogenesis, and cardiac valve formation (15-16,25). In normal adult condition, Wnt protein keeps low level and $\beta$-catenin is degraded by the proteosome (26), whereas Wnt signaling pathway could be activated in pathogenesis status in order to maintain homeostasis $(24,27)$. Data in this study indicated that Endo-MT might participate in the pathogenesis of DCM. Furthermore, we found that Wnt/ $\beta$-catenin pathway was significantly activated in the DCM samples. However, it is difficult to deliberate the increase of Wnt signal coming from Endo-MT-derived myofibroblasts cell in the heart sample of DCM patients. Consistent with our results, Aisagbonhi and co-workers demonstrated that the activation of canonical Wnt signaling is a potentially important regulatory mechanism of Endo-MT-derived myofibroblasts cells, which take part in cardiac tissue repair and myocardial fibrosis after myocardial infarction (8). Collectively, it is plausible to speculate that $\mathrm{Wnt} / \beta$-catenin is probably involved in the process of Endo-MT during the development of DCM.
In addition, transcriptional networks mediating Endo-MT in DCM still remain unclear. Compelling evidence demonstrated that snail might be a crucial factor controlling epithelial-mesenchymal transitions by repressing E-cadherin expression (28). Moreover, previous studies indicated that snail mediated the action of TGF- $\beta 2$ induced Endo-MT $(29,30)$. Endothelial cells that ectopically express snail gene would adopt a fibroblastoid phenotype and acquire invasive properties (31). Our results demonstrated that the protein level of snail was significantly increased in the DCM samples compared with control. Combined with previous studies, our data suggested that snail may play a critical role in Endo-MT during the development of DCM.

During embryogenesis, upregulation of several molecular such as BMP-7 and HGF appeared critical for the reverse of Endo-MT through increasing cadherin expression $(32,33)$. Emerging evidence demonstrated that exogenous recombinant human BMP-7 (rh-BMP) contributed to the regression of Endo-MT in fibrotic tissue (6). Intriguingly, angiotensin II receptor blocker was found to relieve fibrotic progress through blunting Endo-MT in murine models $(9,34)$. Simvastatin treatment decreases TGF- $\beta 1$-induced Endo-MT in vitro (35). These data open up new avenues for the design of specific anti-fibrosis drug via Endo-MT.

In conclusion, our results provide a novel insight that Endo-MT was probably implicated in the pathogenesis of cardiac fibrosis and myocardial remodeling in DCM. Endo-MT might be a potential therapeutic target against cardiac fibrosis and remodeling for DCM treatment. 


\section{Acknowledgements}

The present was supported by grants from National Natural Science Youth Foundation of China (No. 81300166, No. 81300096), 2013 Shanghai Outstanding Academic Leaders Plan (No. 13XD1401500) and Youth foundation of Zhongshan Hospital affiliated to Fudan University (No. 2013ZSQN09).

\section{References}

1. Maron BJ, Towbin JA, Thiene G, Antzelevitch C, Corrado D, Arnett D, Moss AJ, Seidman CE, Young JB; American Heart Association, et al: Contemporary definitions and classification of the cardiomyopathies: An American heart association scientific statement from the council on clinical cardiology, heart failure and transplantation committee; quality of care and outcomes research and functional genomics and translational biology interdisciplinary working groups; and council on epidemiology and prevention. Circulation 113: 1807-1816, 2006.

2. Jellis C, Martin J, Narula J and Marwick TH: Assessment of nonischemic myocardial fibrosis. J Am Coll Cardiol 56: 89-97, 2010.

3. de Leeuw N, Ruiter DJ, Balk AH, de Jonge N, Melchers WJ and Galama JM: Histopathologic findings in explanted heart tissue from patients with end-stage idiopathic dilated cardiomyopathy. Transpl Int 14: 299-306, 2001.

4. Schalla S, Bekkers SC, Dennert R, van Suylen RJ, Waltenberger J, Leiner T, Wildberger J, Crijns HJ and Heymans S: Replacement and reactive myocardial fibrosis in idiopathic dilated cardiomyopathy: Comparison of magnetic resonance imaging with right ventricular biopsy. Eur J Heart Fail 12: 227-231, 2010.

5. Porter KE and Turner NA: Cardiac fibroblasts: At the heart of myocardial remodeling. Pharmacol Ther 123: 255-278, 2009.

6. Zeisberg EM, Tarnavski O, Zeisberg M, Dorfman AL, McMullen JR, Gustafsson E, Chandraker A, Yuan X, Pu WT, Roberts AB, et al: Endothelial-to-mesenchymal transition contributes to cardiac fibrosis. Nat Med 13: 952-961, 2007.

7. Mjaatvedt CH, Lepera RC and Markwald RR: Myocardial specificity for initiating endothelial-mesenchymal cell transition in embryonic chick heart correlates with a particulate distribution of fibronectin. Dev Biol 119: 59-67, 1987.

8. Aisagbonhi O, Rai M, Ryzhov S, Atria N, Feoktistov I and Hatzopoulos AK: Experimental myocardial infarction triggers canonical Wnt signaling and endothelial-to-mesenchymal transition. Dis Model Mech 4: 469-483, 2011.

9. Tang RN, Lv LL, Zhang JD, Dai HY, Li Q, Zheng M, Ni J, Ma KL and Liu BC: Effects of angiotensin II receptor blocker on myocardial endothelial-to-mesenchymal transition in diabetic rats. Int J Cardiol 162: 92-99, 2013.

10. Widyantoro B, Emoto N, Nakayama K, Anggrahini DW, Adiarto S, Iwasa N, Yagi K, Miyagawa K, Rikitake Y, Suzuki T, et al: Endothelial cell-derived endothelin-1 promotes cardiac fibrosis in diabetic hearts through stimulation of endothelial-to-mesenchymal transition. Circulation 121: 2407-2418, 2010.

11. Arciniegas E, Frid MG, Douglas IS and Stenmark KR: Perspectives on endothelial-to-mesenchymal transition: Potential contribution to vascular remodeling in chronic pulmonary hypertension. Am J Physiol Lung Cell Mol Physiol 293: L1-L8, 2007.

12. Selek L, Dhobb M, van der Sanden B, Berger F and Wion D: Existence of tumor-derived endothelial cells suggests an additional role for endothelial-to-mesenchymal transition in tumor progression. Int J Cancer 128: 1502-1503, 2011.

13. Jeong H, Ryu YJ, An J,Lee Y and Kim A: Epithelial-mesenchymal transition in breast cancer correlates with high histological grade and triple-negative phenotype. Histopathology 60: E87-E95, 2012.

14. Zeisberg EM, Potenta SE, Sugimoto H,Zeisberg M and Kalluri R: Fibroblasts in kidney fibrosis emerge via endothelial-to-mesenchymal transition. J Am Soc Nephrol 19: 2282-2287. 2008.
15. Person AD, Garriock RJ, Krieg PA, Runyan RB and Klewer SE: Frzb modulates Wnt-9a-mediated beta-catenin signaling during avian atrioventricular cardiac cushion development. Dev Biol 278: 35-48, 2005.

16. Cohen ED, Tian Y and Morrisey EE: Wnt signaling: An essential regulator of cardiovascular differentiation, morphogenesis and progenitor self-renewal. Development 135: 789-798, 2008.

17. López B, González A, Ravassa S, Beaumont J, Moreno MU, San José G, Querejeta R and Díez J: Circulating biomarkers of myocardial fibrosis: The need for a reappraisal. J Am Coll Cardiol 65: 2449-2456, 2015.

18. Querejeta R, Varo N, López B, Larman M, Artiñano E, Etayo JC, Martínez Ubago JL, Gutierrez-Stampa M, Emparanza JI, Gil MJ, et al: Serum carboxy-terminal propeptide of procollagen type I is a marker of myocardial fibrosis in hypertensive heart disease. Circulation 101: 1729-1735, 2000.

19. Ghosh AK, Bradham WS, Gleaves LA, De Taeye B, Murphy SB, Covington JW and Vaughan DE: Genetic deficiency of plasminogen activator inhibitor-1 promotes cardiac fibrosis in aged mice: Involvement of constitutive transforming growth factor-beta signaling and endothelial-to-mesenchymal transition. Circulation 122: 1200-1209, 2010.

20. Okayama K, Azuma J, Dosaka N, Iekushi K, Sanada F, Kusunoki H, Iwabayashi M, Rakugi H, Taniyama $\mathrm{Y}$ and Morishita R: Hepatocyte growth factor reduces cardiac fibrosis by inhibiting endothelial-mesenchymal transition. Hypertension 59: 958-965, 2012.

21. Khan R and Sheppard R: Fibrosis in heart disease: Understanding the role of transforming growth factor-beta in cardiomyopathy, valvular disease and arrhythmia. Immunology 118: 10-24, 2006.

22. Rivera-Feliciano J, Lee KH, Kong SW, Rajagopal S, Ma Q, Springer Z, Izumo S, Tabin CJ and Pu WT: Development of heart valves requires Gata4 expression in endothelial-derived cells. Development 133: 3607-3618, 2006.

23. Donaldson IJ, Amin S, Hensman JJ, Kutejova E, Rattray M, Lawrence N, Hayes A, Ward CM and Bobola N: Genome-wide occupancy links Hoxa2 to Wnt- $\beta$-catenin signaling in mouse embryonic development. Nucleic Acids Res 40: 3990-4001, 2012.

24. Monga SP: Role of Wnt/ $\beta$-catenin signaling in liver metabolism and cancer. Int J Biochem Cell Biol 43: 1021-1029, 2011.

25. Brade T, Männer J and Kühl M: The role of Wnt signalling in cardiac development and tissue remodelling in the mature heart. Cardiovasc Res 72: 198-209, 2006.

26. Berendsen AD, Fisher LW, Kilts TM, Owens RT, Robey PG, Gutkind JS and Young MF: Modulation of canonical Wnt signaling by the extracellular matrix component biglycan. Proc Natl Acad Sci USA 108: 17022-17027, 2011.

27. Luu HH, Zhang R, Haydon RC, Rayburn E, Kang Q, Si W, Park JK, Wang H, Peng Y, Jiang W and He TC: Wnt/beta-catenin signaling pathway as a novel cancer drug target. Curr Cancer Drug Targets 4: 653-671, 2004.

28. Cano A, Pérez-Moreno MA, Rodrigo I, Locascio A, Blanco MJ, del Barrio MG, Portillo F and Nieto MA: The transcription factor snail controls epithelial-mesenchymal transitions by repressing E-cadherin expression. Nat Cell Biol 2: 76-83, 2000.

29. Niessen K, Fu Y, Chang L, Hoodless PA, McFadden D and Karsan A: Slug is a direct Notch target required for initiation of cardiac cushion cellularization. J Cell Biol 182: 315-325, 2008.

30. Medici D, Potenta S and Kalluri R: Transforming growth factor- $\beta 2$ promotes Snail-mediated endothelial-mesenchymal transition through convergence of Smad-dependent and Smad-independent signalling. Biochem J 437: 515-520, 2011.

31. Lopez D, Niu G, Huber P and Carter WB: Tumor-induced upregulation of Twist, snail and slug represses the activity of the human VE-cadherin promoter. Arch Biochem Biophys 482: 77-82, 2009.

32. Okada $\mathrm{H}$ and Kalluri R: Cellular and molecular pathways that lead to progression and regression of renal fibrogenesis. Curr Mol Med 5: 467-474, 2005. 
33. Okada $\mathrm{H}$ and Kalluri R: Recapitulation of kidney development paradigms by BMP-7 reverses chronic renal injury. Clin Exp Nephrol 9: 100-101, 2005.

34. Tang R,Li Q, Lv L, Dai H,Zheng M, Ma K and Liu B: Angiotensin II mediates the high-glucose-induced endothelial-to-mesenchymal transition in human aortic endothelial cells. Cardiovasc Diabetol 9: 31, 2010.
35. Tuuminen R, Syrjälä S, Krebs R, Keränen MA, Koli K, Abo-Ramadan U, Neuvonen PJ, Tikkanen JM, Nykänen AI and Lemström KB: Donor simvastatin treatment abolishes rat cardiac allograft ischemia/reperfusion injury and chronic rejection through microvascular protection. Circulation 124: 1138-1150, 2011.

(1) (1) This work is licensed under a Creative Commons Attribution-NonCommercial-NoDerivatives 4.0 International (CC BY-NC-ND 4.0) License. 